\title{
Self-assembled nanoscale ferroelectrics
}

\author{
MARIN ALEXE, DIETRICH HESSE \\ Max Planck Institute of Microstructure Physics, D-06120, Halle, Germany
}

\begin{abstract}
Multifunctional ferroelectric materials offer a wide range of useful properties, from switchable polarization that can be applied in memory devices to piezoelectric and pyroelectric properties used in actuators, transducers and thermal sensors. At the nanometer scale, however, material properties are expected to be different from those in bulk. Fundamental problems such as the super-paraelectric limit, the influence of the free surface, and of interfacial and bulk defects on ferroelectric switching, etc., arise when scaling down ferroelectrics to nanometer sizes. In order to study these size effects, fabrication methods of high quality nanoscale ferroelectric crystals have to be developed. The present paper briefly reviews self-patterning and self-assembly fabrication methods, including chemical routes, morphological instability of ultrathin films, microemulsion, and self-assembly lift-off, employed up to the date to fabricate ferroelectric structures with lateral sizes in the range of few tens of nanometers.

(c) 2006 Springer Science + Business Media, Inc.
\end{abstract}

\section{Introduction}

Ferroelectrics are, and will be, widely used in many applications that require sizes down to the nanometer range [1]. It is a challenge to fabricate structures in this range using both lithography ("top-down" approaches) and selfassembly methods ("bottom-up" techniques). Whereas conventional lithographic systems work usually with a resolution of about $100 \mathrm{~nm}$, self-assembly approaches are mainstream methods for the fabrication of structures smaller than $50 \mathrm{~nm}$.

All lithography-based patterning techniques are topdown approaches and represent the basis of nowadays microelectronics technology. They are very well suited to fabricate structures with very good spatial resolution and positioning precision, but are intrinsically limited to structures the sizes of which are well above the lowest nanometer-scale sizes. Thus there is a considerable interest in other fabrication methods which are not based on carving thin films, but rather on building structures from the bottom using atoms and molecules. These bottom-up methods will in principle allow the inexpensive fabrication of structures with sizes of 10 to $20 \mathrm{~nm}$ in a parallel approach. The primary disadvantage of the bottom-up methods is the random positioning of the obtained nanostructures that will make a precise interconnection of them virtually impossible.

Successful strategies and routes have been developed to synthesize nanoscale materials of numerous simple systems such as semiconductors ( $\mathrm{Si}, \mathrm{CdS}$, InAs/GaAs) or 0022-2461 C 2006 Springer Science + Business Media, Inc DOI: $10.1007 / \mathrm{s} 10853-005-5912-\mathrm{x}$ metals. Complex systems such as ferroelectric oxides, or any muticomponent oxides belonging to the class of functional materials, are not systematically addressed so far, in spite of the general possibility to discover a new class of nanomaterials with unique properties. Recently, the first attempts to prepare ferroelectric nanostructures via bottom-up approaches have been published. The approaches can already be classified in two main routes, viz. physical and chemical routes, which are obviously based on different principles. The general characteristics of both routes are the following.

\subsection{Self-patterning via physical routes}

It was recognized that crystallization at all scales, but mostly at nano- and mesoscopic scales, is an important self-assembly strategy in the synthesis and fabrication of small structures. Well-known physical growth concepts, such as island growth, have already been successfully applied to grow nanosize dots of germanium on silicon, or of InAs on GaAs. An important role is played by the lattice mismatch between the substrate and the deposited film. Depositing an epitaxial thin film onto a singlecrystal substrate with high lattice mismatch, the initial stages of the growth process are characterized by either the island (Volmer-Weber) or layer-than-island (StranskiKrastanov) growth modes. In contrast to the layer-bylayer (Frank-van der Merwe) growth mode, which results in a smooth uniform film, the two former growth modes 
are suitable to grow crystalline nano-size dots. The effect is well known and has been carefully studied in the case of simple systems such as germanium on silicon [2], and has even found application in the growth of compound semiconductor lasers [3].

\subsection{Self-patterning via chemical routes}

A number of interesting fabrication approaches appear to be based on chemical routes. Using simple chemical routes it is possible to fabricate nanosize crystals or nanoparticles in colloidal suspensions, which can later be spread onto any substrate surface. After evaporating the solvent, the nanoparticles can be crystallized in two- or even three-dimensional arrays. Although obtaining a regular array of particles is not an easy task, self-assembly concepts based on the reduction of the interfacial energy at the fluid-solid or fluid-fluid interface - concepts that have proven valid for simple systems such as CdS [4] — can be used for more complicated systems such as ferroelectric oxides. One of the most promising routes is based on the microemulsion concept in which a water-in-oil emulsion is produced using a surfactant. Such an emulsion consists of nanometer-size water droplets uniformly dispersed in an oily solvent. This microemulsion can be used to hydrolyse a complex metalorganic precursor. The nanodroplets act as nano-reactors in which the hydrolysis of the precursors takes place and, if the optimum conditions are fulfilled, the final reaction product consists of monodisperse nanosize particles [5].

\section{Self-patterning via physical routes}

\subsection{Self-patterned nano-electrodes}

From the historical point of view the first paper that introduced the concept of ferroelectric nanocells was most probably the paper on nanosize bismuth oxide electrodes obtained by self-assembly [6]. Although not concerned with intentional fabrication, it yet triggered a first concerted effort to obtain and characterize nanoscale ferroelectrics. The authors had observed that during deposition of epitaxial $\mathrm{Bi}_{4} \mathrm{Ti}_{3} \mathrm{O}_{12}$ (BiT) thin films onto silicon substrates, under certain deposition conditions rectangular planar arrays of bismuth-containing crystalline electrodes showing metallic conductivity_-"nano-electrodes"- can be obtained on the surface of the epitaxial BiT thin films (Fig. 1).

The bismuth titanate film was deposited on top of an epitaxial layer of $\mathrm{La}_{0.5} \mathrm{Sr}_{0.5} \mathrm{CoO}_{3}$ (LSCO) on $\mathrm{Si}(100)$, the LSCO layer serving both as an electrode and as an epitaxial template for the ferroelectric film. The nanoelectrodes were uniform and well separated from each other. Depending upon processing conditions the lateral size of the nanoelectrodes was typically $150 \mathrm{~nm}$, yielding an equivalent density of 1 Gbit of ferroelectric cells per chip.

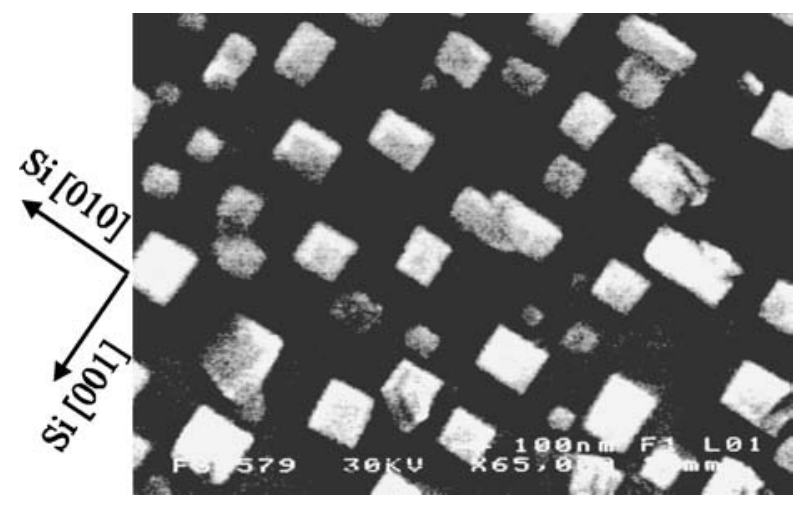

Figure 1 Plan view SEM micrograph of a bismuth-containing nanoelectrode array [6].
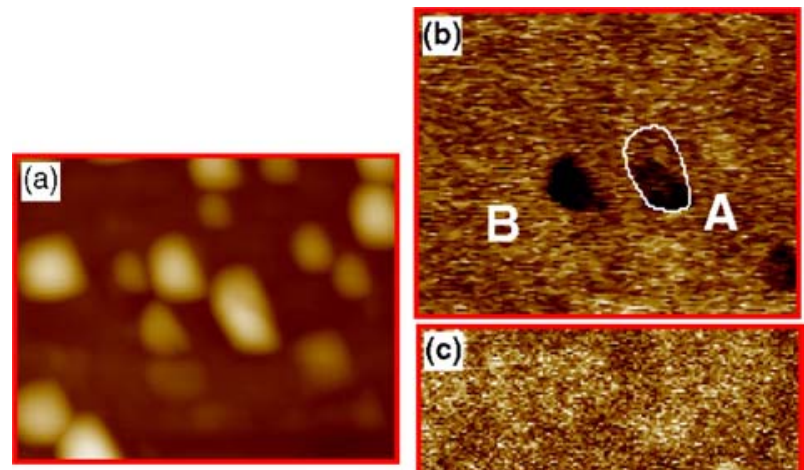

$500 \mathrm{~nm}$

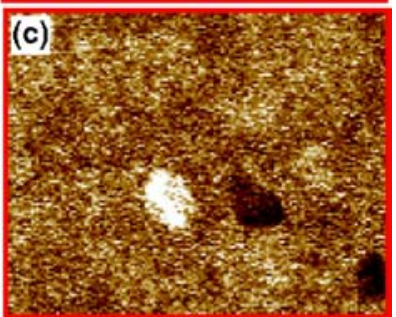

Figure 2 Topography image (a) and piezoelectric images (b, c) showing switching of a single memory cell. The contour of the cell marked "A" has been determined from the topography image. The cell " $\mathrm{B}$ " is shown before (b), and after (c) applying a single voltage pulse of $+10 \mathrm{~V}$ for $100 \mathrm{~ms}$.

The proposed growth mechanism is based on the fact that in bismuth-layered perovskite films any superficial bismuth segregates out as metallic, elemental bismuth at the surface. The deposition taking place in an oxygen atmosphere, the nanoelectrodes were mainly composed of crystalline cubic $\delta-\mathrm{Bi}_{2} \mathrm{O}_{3}$, as determined from high-resolution transmission electron microscopy. Cubic $\delta-\mathrm{Bi}_{2} \mathrm{O}_{3}$ has a defective fluorite structure and is the best ionic conductor known, with a resistivity of only $1 \Omega \mathrm{cm}$ at $1023 \mathrm{~K}$ [7]. Being ionic conductors, the bismuth-containing islands formed mesoscopic capacitors and offered the possibility of locally measuring the ferroelectric properties of the epitaxial c-oriented BiT film. Probing the nanocells via a conductive AFM tip and using piezoresponse force microscopy (PFM), local switching (see Fig. 2) and local hysteresis measurements were performed [8].

This work was significant, because it demonstrated the possibility of decreasing the size of ferroelectric memory cells into the sub-micron range, with a corresponding 

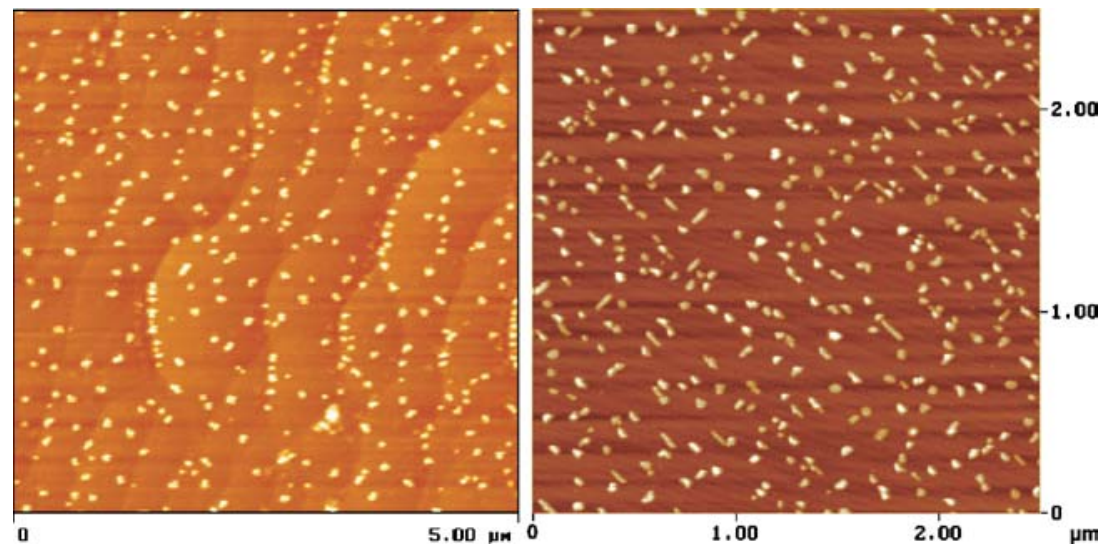

Figure $3 \mathrm{BaTiO}_{3}$ nanostructures deposited onto vicinal $\mathrm{SrTiO}_{3}$ by pulsed laser deposition.

increase of the memory density into the Gbit range, by the time when the commercial and the R\&D prototypes of ferroelectric random access memories (FRAMs) had only kBit densities and were using memory cells of the size of few tens of microns. The self-assembly concept was thus introduced into the fabrication of nanoscale ferroelectrics; however, lateron it was applied to fabricate ferroelectric nanostructures rather than nanoelectrodes.

\subsection{Self-assembled nanostructures by physical vapor deposition}

Very well known methods used for thin film deposition have been among the first methods used to fabricate nanosize structures. These are true self-assembly methods which make use of atoms as building blocks, and of physical growth concepts such as island growth, in order to grow nanosize structures. As it was already pointed out, the main role is played by the mismatch in the lattice constant during the initial stages of the growth process. This mismatch determines different growth modes such as the Stranski-Krastanov or the Volmer-Weber mode, in this way achieving the growth of crystalline nanosize structures instead of a continuous film. Assembling nanostructures is achieved via different mechanisms based on strain fields. This approach can be used to induce a vertical self-assembly, like in the case of germanium islands on silicon, where the germanium islands can "reproduce" themselves in a vertical direction via their strain fields [9]. Based on the same assembly concept, using strain fields generated by pre-patterned [10] or implanted [11] wafers, a lateral self-assembly can also be induced. This concept was successfully applied to simple systems (such as germanium on silicon) and even applied to the preparation of compound semiconductor lasers [3]. However, very little has been done so far-using the above concepts- to obtain nanostructures made from complex oxides. Only recently the growth of complex oxide islands on different substrates using several deposition methods has been achieved. Pulsed laser deposition (PLD) and metalorganic chemical vapor deposition (MOCVD) were the first deposition methods employed.

Pulsed laser deposition and ablation are potential methods to obtain nanoparticles either deposited on a substrate or simply in the form of a dispersed powder. Pulsed laser ablation has been used to produce monodisperse PZT nanoparticles with a fairly narrow size distribution. The experimental setup is rather complex and consists of a laser ablation chamber, a charger, and a furnace. Single crystalline nanoparticles with an average diameter of about $7 \mathrm{~nm}$ and a standard size deviation of $5.4 \%$ were obtained [12]. Using a simple PLD deposition process, Visinoiu et al. have grown $\mathrm{BaTiO}_{3}$ nanostructures on $\mathrm{SrTiO}_{3}$ (STO) substrates [13]. Furthermore, using vicinal STO substrates a certain degree of registration and self-organization can be induced as shown in Fig. 3.

In the case of systems that have a high lattice mismatch, the same concept of island growth leads to the formation of self-assembled structures during the initial growth stages of $\mathrm{SrRuO}_{3}$ (SRO) films on $\mathrm{LaAlO}_{3}$ (LAO) substrates [14]. In the initial growth stage (below a thickness of three monolayers) a periodic ripple forms as a result of stress release via the creation of an additional roughness. Further nucleation is confined to the ripples. As a consequence, at a film thickness of about $2.5 \mathrm{~nm}$, three-dimensional islands assemble on the ripples in a row-like pattern. Due to an anisotropic grain coalescence, most probably originating from an anisotropic diffusion coefficient, a compact regular array of wires results [15]. The nanopatterned SRO films show metallic conductivity and are ferromagnetic (as are bulk films). They show a resistivity of about $280 \mu \Omega \mathrm{cm}$ at room temperature, and a ferromagnetic Curie temperature of about $155 \mathrm{~K}$, slightly lower than the bulk value, due to the presence of stress.

Concerning the growth of nanosize ferroelectric structures employing island growth, the best results so far were most probably obtained using MOCVD. It was found by Shimizu et al., that during initial growth stages of $\mathrm{PbTiO}_{3}$ 

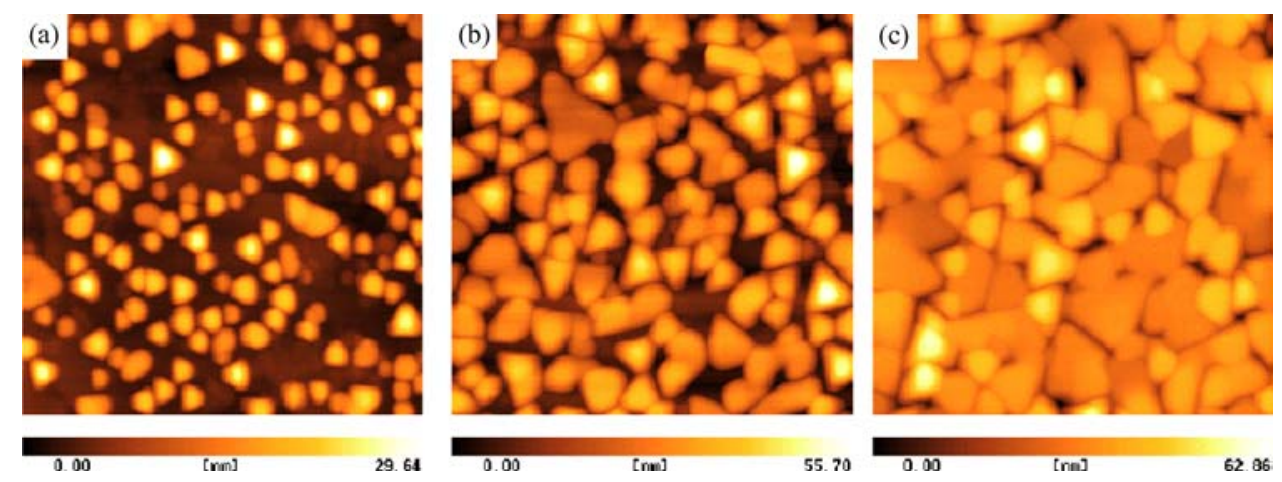

Figure $4 \mathrm{PZT}(\mathrm{Zr} / \mathrm{Ti}=24 / 76)$ island structures deposited for (a) $1 \mathrm{~min}$, (b) $3 \mathrm{~min}$ and (c) $7 \mathrm{~min}$. Scan area is $1 \times 1 \mu \mathrm{m}$. [18].

(PTO) and PZT films on Pt substrates, islands were observed before a continuous film formed [16]. In the case of PZT films, triangular-shaped PZT islands were observed independently of the $\mathrm{Zr} / \mathrm{Ti}$ ratio (Fig. 4). The triangular shape of the PZT islands directly reflects the (111)orientation of the perovskite lattice. A very short deposition time of a few seconds resulted in triangular islands, the size of which increased from $50 \mathrm{~nm}$ to $160 \mathrm{~nm}$ as the deposition time increased from $30 \mathrm{~s}$ to $3 \mathrm{~min}$. At the same time the height increased from 35 to $70 \mathrm{~nm}$, while at longer deposition time the islands coalesced into a continuous film. For a certain deposition time the width and height of the islands decreased as the $\mathrm{Zr}$ content increased. This result corresponds to the fact that a higher growth temperature is required to crystallize $\mathrm{Zr}$-rich PZT than Ti-rich PZT. Transmission electron microscopy (TEM) observations revealed that these PZT islands have tetragonal or rhombohedral structures, and that corresponding PTO islands have a twinned structure showing $90^{\circ}$ domains originating in the tetragonal structure. This was taken as an initial hint to the nanosize island showing ferroelectricity [17].

PFM measurements indeed proved that the MOCVDobtained PZT islands are ferroelectric, but the minimum width and height of the islands that exhibit ferroelectricity were relatively large, viz. 50 and $20 \mathrm{~nm}, 70$ and $30 \mathrm{~nm}$, and 70 and $8 \mathrm{~nm}$ for $\mathrm{Zr} / \mathrm{Ti}$ ratios of $0 / 100,24 / 76$ and $74 / 26$, respectively [18]. The poor ferroelectric behavior was attributed to a degradation of ferroelectricity due to internal stress that is retained in the isolated islands.

An interesting method to pre-define the position of the structures by inducing nucleation sites has been shown by Buhlmann and Muralt $[19,20]$. A $\mathrm{TiO}_{2}$ nucleation site is lithographically pre-defined using electron-beam lithography and etching of a $\mathrm{TiO}_{2}$-coated substrate. The resulted $\mathrm{TiO}_{2}$ dots are only locally increasing the nucleation probability of the PZT and are acting as nucleation sites for the subsequent sputter deposition of PZT. In such way, ferroelectric structures are formed only on the $\mathrm{TiO}_{2}$ dots and not on the remaining free surface. Ferroelectricity in 120-150 nm lateral size structures has been subsequently shown by PFM.

\subsection{Microstructural instability}

One of the most convenient methods to obtain oxide nanoscale structures uses the effect of microstructural instability of ultrathin films. Although this method is frequently named a "chemical route", it is actually a physical self-patterning method. A chemical deposition method is used just to obtain an ultra-thin oxide layer on a substrate of choice, but the self-patterning process itself takes place in the later stage of high-temperature annealing. Briefly, using sol-gel or other metalorganic routes a precursor solution is prepared [21, 22]. This precursor solution is spun on a substrate in order to obtain a thin metalorganic gel film that subsequently is transformed into an amorphous oxide film by pyrolysis, i.e. by a thermal annealing process at a moderate temperature of 250 to $300^{\circ} \mathrm{C}$ in air or oxygen. During the subsequent crystallization anneal, depending on film parameters and annealing conditions, the system transforms either into a normal thin film, or it patterns "itself" into nanostructures of a large variety of shapes and sizes. Seifert et al. [23] were the first to observe microstructural instabilities in perovskite thin films, following previous work on yttria-stabilized zirconia (YSZ) [24]. For example, PTO films on $\mathrm{SrTiO}_{3}$ (STO) substrates, with a nominal film thickness below $100 \mathrm{~nm}$, develop holes during the crystallization anneal (performed in order to convert them into single crystal films). Upon further annealing these holes grow to a stable size, or even cause breaking up of the film into isolated, single crystal islands. In this way relatively thick films entirely cover the substrate, whereas thinner films develop faceted pits and/or holes and finally fall apart into single crystal islands. The above phenomenon is described as a microstructural instability based on the minimization of the global surface energy: During the crystallization anneal, initially isolated pores (that are always present due to the CSD process) grow into surface pits that eventually transform into holes and uncover the substrate. This process depends on the film thickness and pore spacing and implies four different stages [23]. The first stage involves the growth of pyramidal holes until they reach the film-substrate interface. In the second stage the holes continue to grow as truncated pyramids, until they 


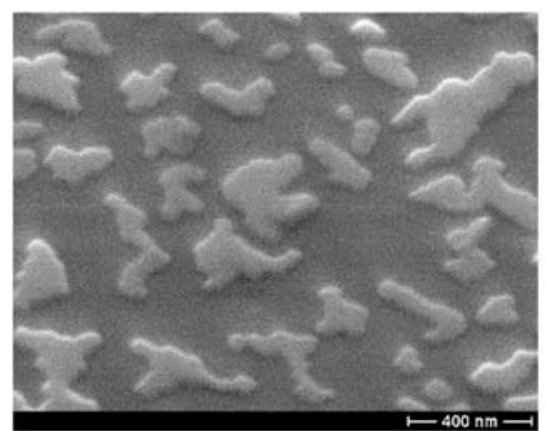

a)

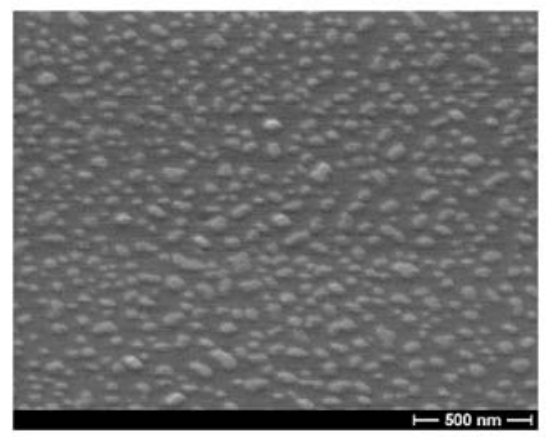

c)

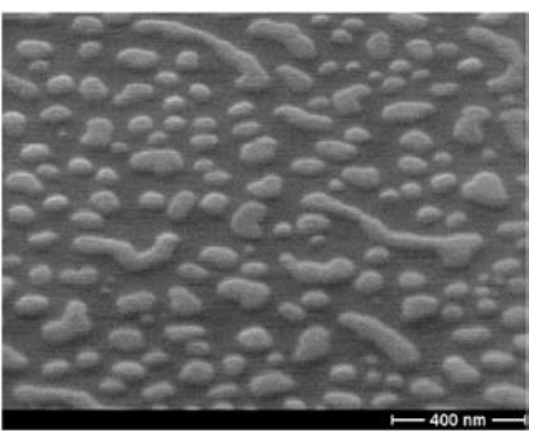

b)

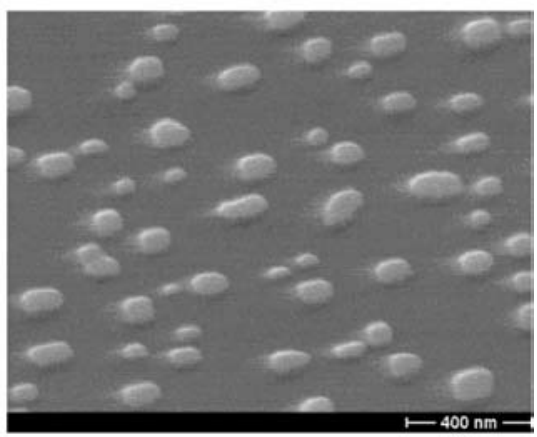

d)

Figure 5 SEM images of PZT nanoislands obtained after deposition of (a) 1:20, (b) 1:25, (c) and (d) 1:40 diluted PZT precursor and after 800 ${ }^{\circ}$ (a-c) and $1100^{\circ} \mathrm{C}(\mathrm{d})$ thermal treatment.

become connected. In the third stage the film is transformed into a collection of truncated pyramids still connected at the lowest edge, until finally the film becomes a collection of unconnected islands in the fourth stage.

The above well-described effect of instability of ultrathin films was used as a "bottom-up way" to fabricate nanoscale ferroelectric structures. Waser et al. obtained polycrystalline PTO nanoscale structures on Pt-coated silicon substrates using a highly diluted precursor [25]. In this way structures with lateral sizes down to $20 \mathrm{~nm}$ were successfully prepared. Using PFM, Roelofs et al. showed that such PTO grains with lateral sizes of about $20 \mathrm{~nm}$ do not exhibit the out-of-plane piezoelectric signal, suggesting that the size at which ferroelectricity vanishes in $\mathrm{PbTiO}_{3}$ is about $20 \mathrm{~nm} \mathrm{[26].}$

If a single crystal substrate is used, the obtained structures are epitaxially oriented as was shown by Szafraniak et al. They obtained epitaxial single crystal ferroelectric structures using the same concept of microstructural instability [27]. Nanosize epitaxial PZT crystals with lateral dimensions of 40 to $90 \mathrm{~nm}$ and a thickness of 9 to $25 \mathrm{~nm}$ were obtained by conventional annealing of ultrathin amorphous oxide films at temperatures above $800^{\circ} \mathrm{C}$ (see Fig. 5). The size, shape and distribution of the nanocrystals could be tuned to some extent by modifying the initial film thickness and the crystallization temperature. The epitaxial nature of the crystals was revealed both by X-ray diffraction analysis and high resolution TEM investigations.

Dawber et al. [28] analyzed the shape and size distribution of both the self-patterned nanoelectrodes and the above PZT nanostructures. They discussed the results of the analysis in terms of island formation mediated by repulsive interactions between the islands and the substrate via strain fields, in a similar way as it had been described for Ge islands on $\mathrm{Si}(100)$ before [29, 30]. Dawber's theory predicts (i) three different kinds of structures such as pyramids, domes, and superdomes, (ii) the volume distribution and (iii) a shape map of the relative population of the structures, both as a function of coverage and crystallization temperature. The self-patterned nanoelectrodes showed a bimodal distribution (see Fig. 6), whereas the shape of PZT structures on STO - due to the very low thickness-transfers from the coexistence of superdomes and domes to the dominance of domes. Apparently this was the first time that the Shchukin-Williams theory was applied to a more complex system than a semiconductor. In addition this approach might offer a practical base for the registration of the islands using strain fields and engineered substrates.

The high quality of the PZT nanocrystals obtained by the above self-assembly method has enabled a detailed structural study performed by Chu et al. [31] on the impact of misfit dislocations on the polarization instability in nanoscale ferroelectrics. The main result of the high 


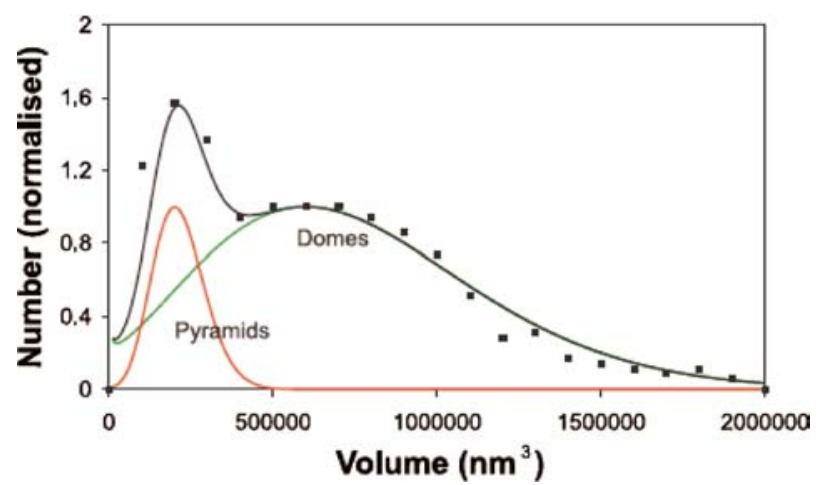

Figure 6 Bimodal distribution of bismuth oxide nanoelectrodes showing the coexistence of pyramids and domes and the excellent fit to the distribution function of Wiliams et al. [30].

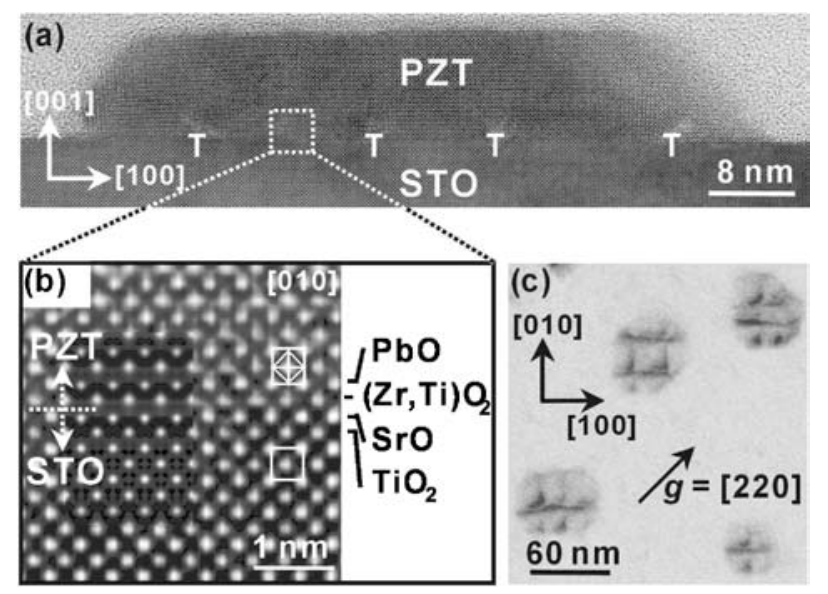

Figure 7 Cross-sectional and plan-view micrographs and calculated contrasts of the PZT (48/52) nanocrystals. (a) Cross-sectional HREM image of a (001)-oriented PZT nanoisland in [010] projection; misfit dislocations indicated by T. (b) An enlarged interface zone of a; the rectangle and square indicating respective PZT and STO lattices; inset, multislice contrast simulation at $t=4 \mathrm{~nm}$ and $\Delta f=-60 \mathrm{~nm}$ with $\mathrm{PbO}-(\mathrm{Zr}, \mathrm{Ti}) \mathrm{O}_{2}-\mathrm{SrO}_{-} \mathrm{TiO}_{2}$ stacking across the interface. At these imaging conditions, bright and dark contrasts represent the cation and anion columns, respectively. (c) Bright-field planview image recorded under $g=[220]$ on specimens annealed at $950^{\circ} \mathrm{C}$ for $1 \mathrm{~h}$, the dark contrasts showing the network of misfit dislocations [31].

resolution TEM investigations was the observation of a high-strain region formed around each misfit dislocation (Fig. 7). (Such misfit dislocations generally occur to relax the strain in epitaxially grown structures and films). For the studied case of PZT (48/52) nanoislands grown on STO substrates, the cross section of the highly distorted "tube" around each dislocation is about $8 \mathrm{~nm}$ in width and $4 \mathrm{~nm}$ in height. In this volume the tetragonal lattice is distorted due to triaxial strain fields, so that the ferroelectric polarization of an island with a height of about $9 \mathrm{~nm}$ and a lateral size of $20 \mathrm{~nm}$ becomes unstable. This was interpreted as a size effect which actually originates from an extrinsic effect (viz. the misfit dislocations) rather than from the super-paraelectric limit. It was also shown that avoiding the formation of misfit dislocations at the inter- face, the ferroelectricity could be retained in very small structures of this type.

The above studies have clearly shown that on the one hand high-quality structures approaching ideal systems are required to evidence true size effects, and that on the other hand single defects in these nanoscale structures can be most detrimental for the ferroelectric properties. In other words, the extrinsic size effects generated by interfaces, surfaces, strain, compositional inhomogeneities, etc. can have a higher negative impact on the polarization stability than intrinsic size effects.

\section{Self-patterning via chemical routes \\ 3.1. Hydrothermal growth}

Hydrothermal synthesis is a unique technique for the low-temperature fabrication of powders or films. It involves the growth of oxides from an aqueous solution at moderate temperatures (about $100-400^{\circ} \mathrm{C}$ ) and pressures (0.1-15 MPa). During the last decade this method was applied to produce various oxides at low temperatures [32]. Perovskite ferroelectrics can be easily synthesized by this technique at temperatures below $200^{\circ} \mathrm{C}$. For instance, barium titanate was grown even at $90^{\circ} \mathrm{C}$ without any additional post-deposition thermal treatment [33-35]. Hydrothermal growth can be used as a bottom-up strategy in order to obtain complex oxide nanoscale structures. Usually, one needs to mix an equimolar amount of precursors in an aqueous solution with a very high $\mathrm{pH}$ value, and subject the solution for a certain time to a low-temperature annealing under pressure. For example, appropriate precursors for $\mathrm{Ti}, \mathrm{Pb}$, and $\mathrm{Ba}$ ions could be $\mathrm{TiO}_{2}, \mathrm{~Pb}\left(\mathrm{NO}_{3}\right)_{2}$, and barium acetate, respectively. The $\mathrm{pH}$ value of the solution plays a very important role to stabilize the desired phase. For the $\mathrm{Ba}-\mathrm{Ti}-\mathrm{H}_{2} \mathrm{O}-\mathrm{CO}_{2}$ system, barium titanate is stable for a $\mathrm{pH}>12$ [36].

Recently Szafraniak et al. obtained ferroelectric epitaxial nanocrystals using a hydrothermal route [37]. As expected, besides the initial stoichiometry and the $\mathrm{pH}$ value, temperature and time play an important role for the obtained structures. For $\mathrm{PbTiO}_{3}$ grown on (111) STO the first visible crystals nucleate after $2 \mathrm{~h}$ while at $150^{\circ} \mathrm{C}$ the first pyramid-shaped structures appear after $4 \mathrm{~h}$ of treatment. For a longer time the nuclei grow and eventually coalesce in larger structures or films. As expected, the coalescence process takes longer time at lower temperatures. Interestingly, in few cases a self-assembled, fairly ordered arrangement was observed (Fig. 8). This fact is most probably due to the substrate preparation (e.g., the polishing process) which could induce a spatial arrangement of nucleation centers, for instance along steps of vicinal surfaces. This suggests that a certain degree of orientation and order can be induced, manipulating the nucleation process at the substrate surface. For instance, a pre-patterning or a deposition of monolayers of materials 


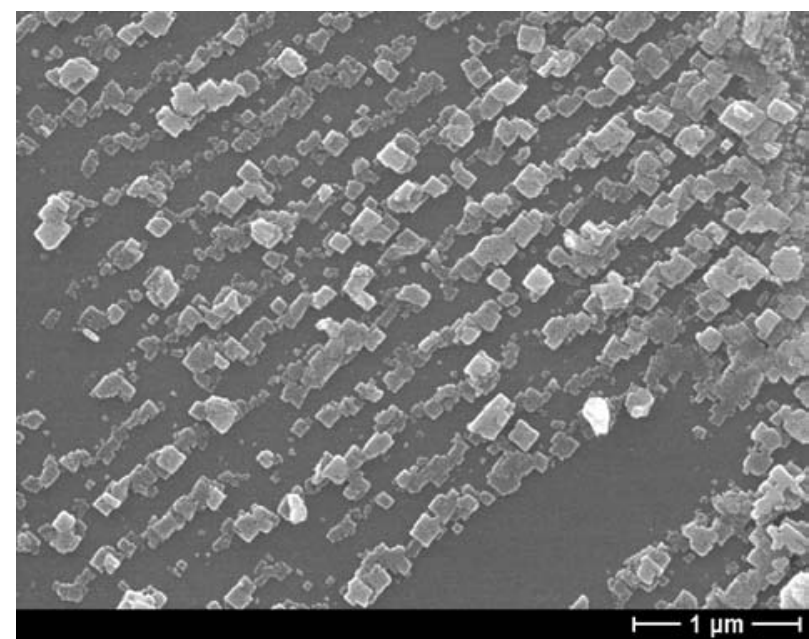

Figure 8 Highly ordered epitaxial PZT nanostructures obtained by hydrothermal growth on $\mathrm{Nb}$ :STO (100) single crystal substrates [37].

with different wetting properties might induce a preferential nucleation and registration of the obtained structures.

\subsection{Synthesis of nanoparticles}

Provided that there will be appropriate methods to manipulate nanosize particles and to deposit them into monolayers on desired substrates, or even to arrange them in a registered way, the fabrication of nanoparticles via chemical routes might be of high interest. This interest should be due to the flexibility of the process of low-temperature processing by which very small nanosize particles made from complex materials can be obtained.

Vapor phase routes, such as vapor deposition (CVD) and pulsed laser deposition (PLD) are suitable to produce high-purity nanoparticles, but suffer from the high cost of equipment and from low yield. The solution-based chemical routes potentially offer better grain-size homogeneity and good stoichiometry control. A number of routes to produce fine powders have already been established. These include co-precipitation, sol-gel processing, hydrothermal synthesis, and other routes.

Two different approaches are considered in order to synthesize nanoparticles via chemical routes. One is the preparation of stabilized particles dispersed in liquids, and the second is an in-situ preparation using a surfactant assembly such as Langmuir-Blodgett films, micelles, microemulsions, or similar, all based on colloid chemistry. In all cases the desired output is represented by nanometer size particles dispersed in a solvent. The stability of the solution is given by the balance between the short range attractive forces among the particles and the repulsive forces [38]. The obtained particles can be transferred to a substrate using an appropriate technique such as the Langmuir-Blodgett method. Appropriate control of the particles' hydrophobicity, surface charge and charge density can result in monolayers of particles that later can be transferred to a solid substrate [39].

\subsubsection{Sol-gel synthesis}

Sol-gel synthesis is one of the most common routes to synthesize oxide particles, in particular particles of ferroelectric oxides. The synthesis follows a simple route, starting with the preparation of a complex alkoxide chosen according to the final formula of the desired oxide compound. The obtained alkoxyde is then hydrolyzed in a controlled way to obtain a precipitate. Finally the nano-particle precipitate is washed and dispersed in a certain solvent. To obtain a stable suspension from the nano-particles is the most difficult part of the process. A successful attempt to synthesize stable monodisperse nanosize particles using a simple sol-gel method was recently reported by Liu et al. [40]. They prepared a complex metalorganic compound from lead acetate, Ti ethoxide and $\mathrm{Zr}$ butoxide in pure acetic acid in order to avoid any reaction with water. The solution was slowly precipitated at $80^{\circ} \mathrm{C}$ by adding ammonia via an argon bubbler, and then kept at this temperature for several days to allow the particles to grow. The dried product of nanoparticles, which were initially amorphous, was crystallized at temperatures ranging from $400^{\circ}$ to $800^{\circ} \mathrm{C}$ in argon atmosphere and then in oxygen at a lower temperature. A stable suspension can be formed after modifying the surface of the nanoparticles with tartrate ligands. Free standing nanoparticles of $10 \mathrm{~nm}$ to $30 \mathrm{~nm}$ diameter were prepared in this way.

Two key steps allow the preparation of free standing crystalline nanoparticles: (i) the annealing in argon which - unlike annealing in oxygen - prevents sintering of the nanoparticles, and (ii) a chemical treatment of the surface (in this case with a tartrate ligand) which allows the suspension in a solution. As it will be seen later, the last step is decisive to achieve a suspension of free-standing particles, because un-coated particles are usually quickly aggregating and precipitating.

A slightly different route was implemented by O'Brian et al. [41] to prepare nanosize $\mathrm{BaTiO}_{3}$ particles. A single bimetallic alkoxide precursor (barium titanium ethylhexano-isopropoxide) was injected into a mixture of diphenylether and oleic acid as stabilizing agent at $140^{\circ} \mathrm{C}$ under an inert atmosphere. After distillation of 2-propanol the resulting solution was cooled to $100^{\circ} \mathrm{C}$, and hydrogen peroxide was injected. The solution was maintained at this temperature for $48 \mathrm{~h}$ to promote hydrolysis and crystallization under the conditions of inverse micelles. Monodisperse $\mathrm{BaTiO}_{3}$ particles of 4 to $8 \mathrm{~nm}$ diameter were obtained. The diameter could be tuned modifying the ratio between alkoxide precursor and oleic acid, which also passivated the surface of the particles, in this way preventing the agglomeration and enabling the transfer of the 


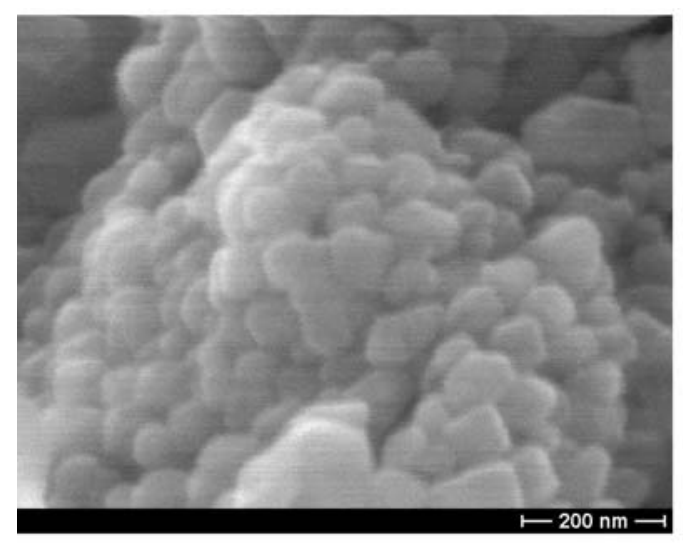

(a)

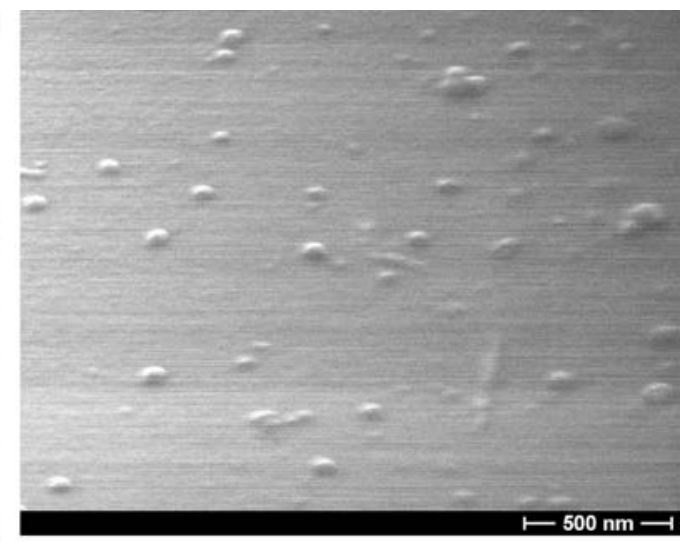

(b)

Figure 9 Scanning electron microscopy image of (a) agglomerated $\mathrm{BaTiO}_{3}$ nanoparticles and (b) $\mathrm{BaTiO}_{3}$ nanoparticles deposited on a $\mathrm{Nb}$ :STO substrate.

particles into a nonpolar solvent. Two points are remarkable in this route. First, the crystalline phase occurs at temperatures as low as $100^{\circ} \mathrm{C}$ directly from the reaction, and second the reaction takes place in a so called inverse micelle, i.e. a colloidal-type solution. The colloidal or microemulsion route is a generic route to synthesize nanoparticles as discussed in the following.

\subsubsection{Generation of nanoparticles in microemulsions}

Among all the chemical routes, the microemulsion technique is the most promising method to prepare nanoscale perovskite particles. The basic characteristic of this route is the confinement of the involved reactions in so-called "nanoreactors". The particle formation in nanoreactors takes place simultaneously in a number of about $10^{18}$ $10^{20}$ nano-compartments separated from each other [42]. The microemulsion technique has already been used to prepare ultrafine powders from a variety of materials, including metals [43], metal oxides [44], and high- $\mathrm{T}_{\mathrm{C}}$ $\mathrm{YBa}_{2} \mathrm{Cu}_{3} \mathrm{O}_{7-x}$ superconductors [45]. Herrig and Hempelmann [5] were among the first to use the microemulsion approach for the preparation of ultrafine powders of simple perovskites. A comprehensive review on the preparation of nanosize oxides via microemulsion-mediated synthesis is given by Osseo-Asare [46].

It is not the goal of the present paper to review this route in detail. We would just like to point out the possibilities of the microemulsion route and to show a few examples. Briefly, a miniemulsion or microemulsion is a system where small droplets of high stability are created using high shear [42]. The system must have at least three components, viz. a continuous phase, a dispersed phase, and a surfactant. An unstable system is, e.g., given by a water-in-oil system which is simply obtained by mixing a dispersed phase (water) in the continuous phase (oil). The stability of such a system is obtained by adding an agent that dissolves a dispersed phase but is insoluble in the continuous phase. For a water-in-oil system, an oil, water, an emulsifier (surfactant) and a hydrophobic agent are mechanically homogenized (using, e.g., ultrasonic agitation) to obtain monodisperse droplets in the size range between 30 and $500 \mathrm{~nm}$. A simple water-in-oil system is considered being unstable due to two droplet growth mechanisms, Ostwald ripening and coalescence. The surfactants control the coalescence, while the Ostwald ripening is controlled by the hydrophobic agent. Moreover, the type of surfactant used for the stabilization can be used to adjust the size of the droplets.

The nanodroplets of a water-in-oil system are an ideal medium for the preparation of very fine oxide particles, either by a sol-gel type hydrolysis of metal alkoxides in the microemulsion-provided nano-reactors, or by coprecipitation of an aqueous solution of mixed oxalates or nitrates from the corresponding microemulsion. According to Beck et al. [47], nanoscale $\mathrm{BaTiO}_{3}$ particles can be fabricated using the sol-gel-type hydrolysis approach. First a microemulsion is prepared by mixing a surfactant (Tergitol TNP-35, TNP-10, or TNP-7) with cyclohexane and 1-octanol, adding water. The resulting microemulsion can then be used to hydrolyze $\mathrm{a} \mathrm{BaTiO}_{3}$ alkoxide precursor to yield fine $\mathrm{BaTiO}_{3}$ particles with a crystallite size tailored in a broad range from $3 \mathrm{~nm}$ to about $60 \mathrm{~nm}$, each size having a relatively narrow distribution. The particle diameter is nicely tuned by adjusting the droplet size of the microemulsion, knowing that the latter is determined by the length of the hydrophilic part of the surfactant. This effect was used by Beck et al. to tune the crystallite size of $\mathrm{BaTiO}_{3}$ performing a hydrolysis of a complex alkoxide in different microemulsions prepared with different surfactants.

An important advantage of the microemulsion route is the low calcination temperature required for the formation of the perovskite phase, which is up to $200^{\circ} \mathrm{C}$ lower than in the conventional solid-state reaction methods [48]. Even 


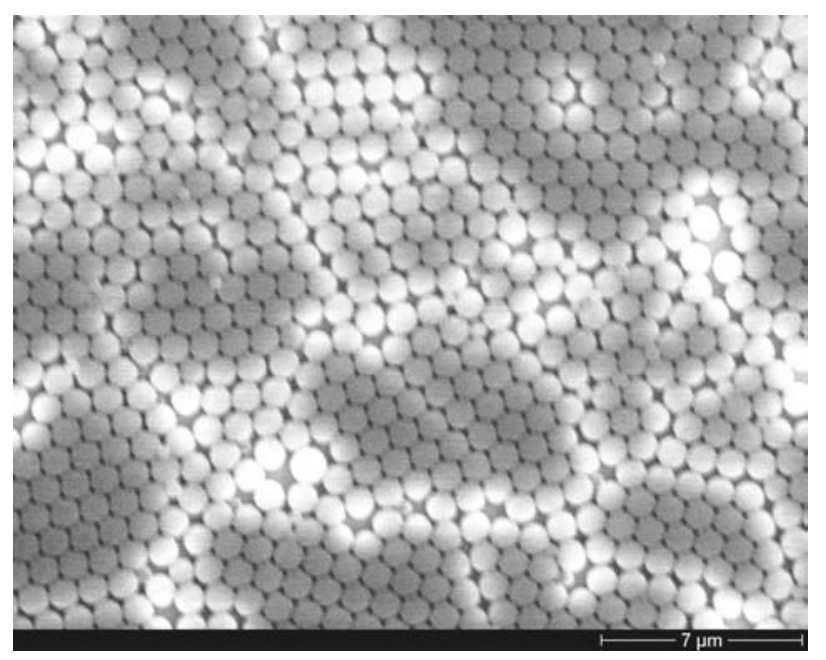

Figure 10 SEM image of a self-assembled monolayer of monodisperse latex spheres of $1 \mu \mathrm{m}$ diameter.

the formation of a pure barium titanate perovskite was reported during the wet chemical precipitation followed by any calcination treatment [5].

Bhattacharyya et al. [49] have used a microemulsion route to produce monodisperse nanoparticles, i.e. particles dispersed in a liquid medium, and to obtain $\mathrm{BaTiO}_{3}$ structures of 50-60 nm diameter in a random distribution on $\mathrm{Nb}$ :STO substrates, as shown in Fig. 9.

\section{Self-assembled lithography}

An economic, versatile means to fabricate arrays of ferroelectric nanostructures is given by the possibility to use a self-assembled lift-off deposition mask in combination with a physical or chemical vapour deposition technique. For example, a principal possibility is the combination of a self-assembled monolayer mask of hexagonally close-packed monodisperse latex spheres with the physical vapour deposition of an array of metal nanodots, called "Natural lithography", "Nanosphere lithography" or "Shadow nanosphere lithography" [50-52]. This technique can also be applied to the fabrication of ferroelectric nanostructure arrays, as has been demonstrated by Ma and Hesse [53-55].

A self-assembled monolayer of monodisperse latex spheres arranged in a close-packed hexagonal array was prepared according to [51, 52] and was used as a lift-offtype deposition mask for pulsed laser deposition (PLD) of $\mathrm{BaTiO}_{3}$ (BTO) or $\mathrm{SrBi}_{2} \mathrm{Ta}_{2} \mathrm{O}_{9}$ (SBT) nanostructures [5355]. BTO and SBT were studied as prototypes for perovskite and bismuth-layered perovskite ferroeletric materials, respectively. The latex-sphere monolayer was prepared on a conductive (100)-oriented $\mathrm{Nb}$-doped $\mathrm{SrTiO}_{3}$ single crystal substrate (that later served as a bottom electrode) by a spin-coating process, using commercial monodisperse polystyrene latex sphere dispersions with spheres of $1 \mu \mathrm{m}$ or $0.5 \mu \mathrm{m}$ diameter. Fig. 10 is a scanning electron micrograph (SEM image) of such a monolayer. The bright regions correspond to stacking faults within the layer. Avoiding the formation of stacking faults is possible, e.g. on an area of $50 \mu \mathrm{m}^{2}$ [56]. This requires, however, some effort, skill and experience, cf. [56]. Stacking faults can, however, also made use of. They provide somewhat larger spacings for deposition, resulting in larger than usual lateral sizes of the prepared ferroelectric structures. These larger structures may be used to compare their physical properties with those of the regular, small nanostructures, as shown below.

Pulsed laser deposition of BTO and SBT followed by the lift-off of the polystyrene latex spheres in methylene chloride, and the subsequent crystallization anneal, yield a regular pattern of ferroelectric nanostructures as shown in Fig. 11.

The advantage of such a technique is that-using appropriate substrates - the nanostructures can be epitaxially crystallized $[54,55]$. Moreover, using piezoresponse scanning force microscopy (PFM) the structures can be accurately characterized by measuring piezoelectric hysteresis loops (see Fig. 12). This enabled a detailed study of - and revealed a thickness-dependent imprint effect in

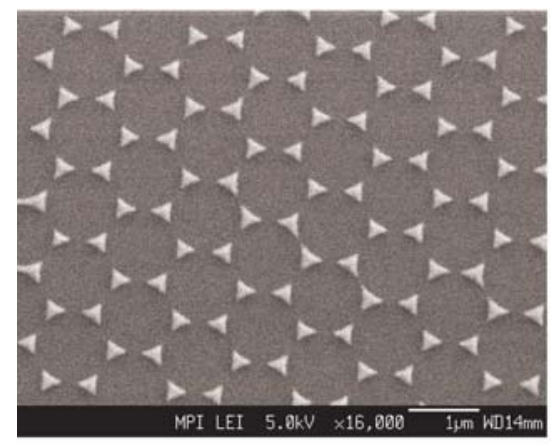

(a)

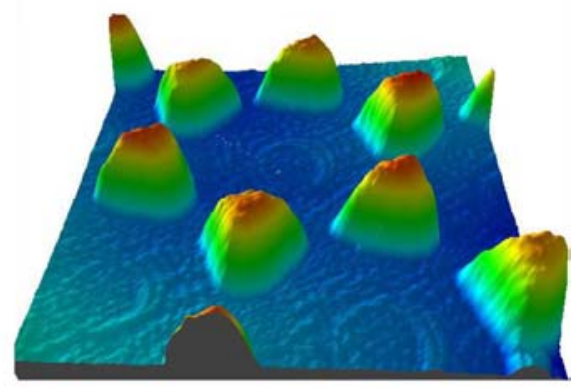

(b)

Figure 11 (a) SEM image and (b) Scanning force (AFM) topography image $(2 \times 2 \mu \mathrm{m})$ of part of a $\mathrm{SrBi}_{2} \mathrm{Ta}_{2} \mathrm{O}_{9}$ array fabricated from a self-assembled monolayer of latex spheres of $1 \mu \mathrm{m}$ diameter. 


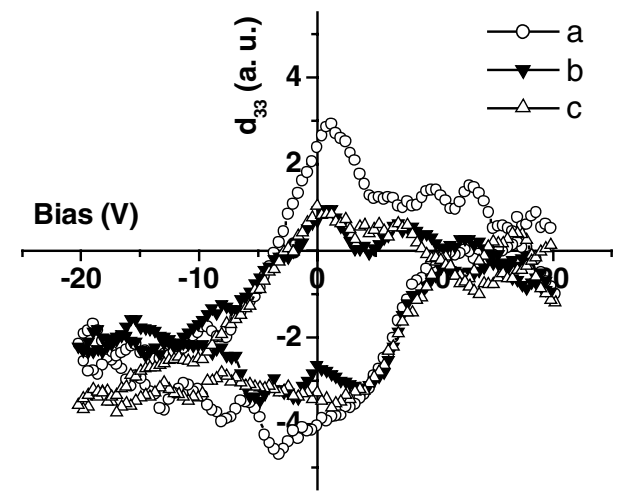

Figure 12 PFM hysteresis loops obtained from BTO nanostructures of different size. $d_{33}$ is the effective piezoelectric coefficient along the vertical of the nanostructure. For curves a, b, and c, see the text.

- both the BTO and the SBT nanostructures. For example, Fig. 12 shows that the hysteresis curve (a) of a $44 \mathrm{~nm}$ high BTO nanostructure (fabricated from a $1 \mu \mathrm{m}$ sphere mask) is more or less symmetric, whereas the loops (b) and (c) of two only $26 \mathrm{~nm}$ high nanostructures (fabricated using a $0.5 \mu \mathrm{m}$ sphere mask) are shifted towards the negative $\mathrm{d}_{33}$ axis, i.e. are imprinted. This effect is independent of the lateral size, as is also shown in the figure: Curve (c) was recorded from a laterally extended structure, that had been formed at a stacking fault of the sphere mask, whereas curve (b) is from a laterally small structure.

In cases where three-dimensional nanostructures have clearly developed lateral surfaces, a similar sizedependent imprint effect may occur [57]. To explain this effect, as well as the thickness-dependent imprint effect observed in the laser-deposited arrays shown above, a phenomenological model has been developed. According to this model, the imprint effect in the flat $\mathrm{BaTiO}_{3}$ nanostructures results from a domain locking near the ferroelectricelectrode interface, so that the nanostructures are composed of switchable regions and of non-switchable ones, the latter being close to the interface with the substrate. A similar effect occurs at the lateral surfaces of the threedimensional nanostructures.

In summary, self-assembled lithography methods based on latex monolayers permit a rapid, cost-effective fabrication of high-quality ferroelectric nanostructures by physical or chemical vapor deposition enabling in-depth studies of size effects in nanoscale ferroelectrics.

\section{Conclusions}

The successful preparation of sub-micron and nanosize ferroelectric structures and the development of appropriate nanoscale measurement methods have opened a new emerging field, viz. nanoscale ferroelectrics. Bottom-up approaches offer the possibility of a low-cost fabrication of ferroelectric structures with lateral sizes of 10 to $50 \mathrm{~nm}$, well below the sizes accessible by any of the top-down methods on relatively large substrates. Unfortunately, registration of the structures is still a major problem of all bottom-up approaches, and quite an effort will be necessary to solve this problem. Some of the self-assembly methods offer the possibility to fabricate high-quality epitaxial or single crystalline nanostructures, which represents a major advantage in addressing fundamental problems such as size effects, domain pinning at nanoscale, or determination of the super-paraelectric limit. In any case suitable fabrication methods to obtain high-quality nanoscale ferroelectrics are now available, which make us assert that nanoscale ferroelectrics will be part of our near future.

\section{References}

1. O. AUCiello, J. F. SCOTT and R. RAMESh, Physics Today 51 (1998) 22.

2. D. J. EAGLeShAM and M. CERULlo, Phys. Rev. Lett. 64 (1990) 1943.

3. R. L. SEllin, C. RiBBAT, M. GRUNDMANN, N. N. LEDENTSOV and D. BIMBERG, Appl.Phys. Lett. 78 (2001) 1207.

4. Y. LIN, H. SKAFF, T. EMRICK, A. D. DINSMORE and T. P. RUS SELL, Science 299 (2003) 226.

5. H. HERRIG and R. HEMPELMANN, Mater. Lett. 27 (1996) 287.

6. M. ALEXE, J. F. SCOTT, C. CURRAN, N. D. ZAKHAROV, D. HESSE and A. PIGNOLET, Appl. Phys. Lett. 73 (1998) 1592.

7. T. TAKAHASHI and H. IWAHARA, Mater. Res. Bull. 13 (1978) 1447.

8. M. Alexe, A. GRuverman, C. HAmAgeA, N. D. ZAKHAROV, A. PignOlet, D. HeSSE and J. F. SCOTT, Appl. Phys. Lett. 75 (1999) 1158.

9. O. G. SCHMIDT and K. EBERL, Phys. Rev. B-Condensed Matt. 61 (2000) 13721.

10. O. G. SCHMIDT, U. DENKER, K. EBERL, O. KIENZLE, F. ERNST and R. J. HAUG, Appl. Phys. Lett. 77 (2000) 4341.

11. H. OMI, D. J. BOTTOMLEY, Y. HOMMA and T. OGINO, Phys. Rev. B-Condensed Matt. 67 (2003) 115302.

12. K. S. SEOL, S. TOMITA, K. TAKEUCHI, T. MIYAGAWA, T. KATAGIRI and Y. OHKI, Appl. Phys. Lett. 81 (2002) 1893.

13. A. VISINOIU, Private communication (2001).

14. E. VASCO, R. DITTMANN, S. KARTHAUSER and R. WASER, Appl. Phys. Lett. 82 (2003) 2497.

15. S. KARTHAUSER, E. VASCO, R. DITTMANN and R. WAS ER, Nanotechnology 15 (2004) S122.

16. M. SHIMIZU, M. SUGIYAMA, H. FUJISAWA, T. HAMANO, T. SHIOSAKI and K. MATSUSHIGE, J. Cryst. Gr. 145 (1994) 226.

17. H. FUJISAWA, K. MORIMOTO, M. ShimizU, H. NiU, K. HONDA and S. OHTA Ni, Japan Soc. Appl. Phys. Japanese J. Appl. Phys. Part 1-Regular Papers Short Notes \& Review Papers 39 (2000) 5446.

18. F. FUJISAWA, K. MORIMOTO, M. ShimizU, H. NIU, K. HONDA and S. OHTA NI, Ferroelectric Thin Films IX. Symposium (Materials Research Society Symposium Proceedings Vol.655). Mater. Res. Soc. (2001) CC10.

19. P. MURALT, S. BUHLMANN and S. VON ALLMEN, Mater. Res. Soc. Symposium Proceedings 784 (2004) 13.

20. S. Buhlmann, P. MURAlt and S. VOn Allmen, Appl. Phys. Lett. 84 (2004) 2614.

21. K. D. BUDD, S. K. DEY and D. A. PAYNE, Brit. Ceram. Proc. (1985) 107.

22. T. SCHNELLER and R. WASER, Ferroelectrics 267 (2002) 293. 
23. A. SEIFERT, A. VOJTA, J. S. SPECK and F. F. LANGE, J. Mater. Res. 11 (1996) 1470.

24. K. T. MILLER, F. F. LANGE and D. B. MARSHALL, ibid. 5 (1990) 151.

25. R. WASER, T. SCHNELLER, S. HOFFMANN-EIFERT and P. EHRHART, Integ. Ferroelect. 36 (2001) 3.

26. A. ROELOFS, T. SCHNELLER, K. SZOT and R. WASER, IOP Publishing. Nanotechnology 14 (2003) 250.

27. I. SZAFRANIAK, C. HARNAGEA, R. SCHOLZ, S. BHATTACHARYYA, D. HESSE and M. AlEXE, Appl. Phys. Lett. 83 (2003) 2211.

28. M. DAWBER, I. SZAFRANIAK, M. ALEXE and J. F. SCOT T, J. Phys.-Condensed Matter 15 (2003) L667.

29. V. A. SHCHUKIN, N. N. LEDENTSOV, P. S. KOPEV and D. BIMBER G, Phys. Rev. Lett. 75 (1995) 2968.

30. R. S. Williams, G. MEDEIROS-RIBEIRO, T. I. KAMINS and D. A. A. OHLBERG, Ann. Rev. Phys. Chem. 51 (2000).

31. M. W. CHU, I. SZAFRANIAK, R. SCHOLZ, C. HARNAGEA, D. HESSE, M. ALEXE and U. GOSELE, Nat. Mater 3 (2004) 87

32. F. F. LANGE, Science 273 (1996) 903.

33. A. T. CHIEN, J. S. SPECK and F. F. LANGE, J. Mater. Res. 12 (1997) 1176.

34. A. T. CHIEN, J. S. SPECK, F. F. LANGE, A. C. DAYKIN and C. G. LEVI, ibid. 10 (1995) 1784.

35. A. T. CHIEN, L. ZHAO, M. COLIC, J. S. SPECK and F. F. LANGE, ibid. 13 (1998) 649.

36. P. BENDALE, S. VENIGALLA, J. R. AMBROSE, E. D. VERINK, JR. and J. H. ADAIR, J. Amer. Ceram. Soc. 76 (1993) 2619.

37. I. SZAFRANIAK and M. ALEXE, Ferroelectrics 291 (2003) 19.

38. D.F. EVANS and H. WENNERSTROM, in "The Colloid Domain. Where Physics, Chemistry and Technology Meet" (Wiley-VCH, New York, 1994).

39. N. A. KOTOV, F. C. MELdRUN and J. H. FENDLER, J. Phys. Chem. 98 (1994) 8827.
40. C. LIU, B. ZOU, A. J. RONDINONE and J. Z. ZHANG, J. Amer. Ceram. Soc. 123 (2001) 4344

41. S. O'BRIEN, L. BRUS and C. B. MURRAY, J. Amer. Chem. Soc. 123 (2001) 12085.

42. K. LANDFESTER, Adv. Mater. 13 (2001) 756.

43. F. JIYE, K. L. STOKES, J. WIEMANN and Z. WEILIE, Mater. Lett. 42 (2000) 113.

44. S. BANDOW, K. KIMURA, K. KON-NO and A. KITAHARA, Japanese J. Appl. Phys. Part 1-Regular Papers Short Notes \& Review Papers 26 (1987) 713.

45. P. AYYUB, A. N. MAITRA and D. O. SHAH, Physica $C 168$ (1990) 571.

46. K. OSSEO-ASS ARE, in "Handbook of Microemulsion Science and Technology", edited by P. Kumar and K. L. Mittal (Marcel Dekker, Inc., New York, Basel, 1999) p. 549.

47. C. BECK, W. HARTL and R. HEMPELMANn, J. Mater. Res. 13 (1998).

48. J. WANG, J. FANG, S.-C. NG, L.-M. GAN, C. H. CHEW, X. WANG and Z. SHEN, J. Amer. Ceram. Soc. 82 (1999) 873.

49. S. BHATTACHARYYA, S. CHATTOPADHYAY and $\mathrm{M}$. ALEXE, Nanomaterials for Structural Applications. Symposium (Mater. Res. Soc. Symposium Proceedings Vol. 740). Mater. Res. Soc. (2003) 333.

50. A. KORIOSEK, W. KANDULSKI, P. CHUDZINSKI, K. KEMPA and M. GIER SIG, Nano Lett. 4 (2004) 1359.

51. H. W. DECKMAN and J. H. DUNSMUIR, Appl. Phys. Lett. 41 (1982) 377

52. J. C. HULTEEN and R. P. VAN DUYNe, J. Vac. Sci. Technol. A 13 (1995).

53. W. MA and D. HESSE, Appl. Phys. Lett. 84 (2004) 2871.

54. W. MA, C. HARNAGEA, D. HESSE and U. GOSELE, ibid. 83 (2003) 3770

55. W. MA and D. HES SE, ibid. 85 (2004) 3214.

56. J. RYBCZYNSKI, U. EBELS and M. GIERSIG, Coll. Surf. A: Physicochem. Eng. Asp. 219 (2003) 1.

57. M. AlEXE, C. HARnAGEA, D. HESSE and U. GOSELE, Appl. Phys. Lett. 79 (2001) 242. 